\title{
A Framework of Information Technology for Water Resources Management
}

\author{
P.S.V.S. Sridhar \\ University of Petroleum \\ \& Energy Studies \\ Dehradun
}

\author{
T. N. Jowhar \\ Wadia Institute of \\ Himalyan Geology, \\ Dehradun
}

\author{
Anant Bhaskar Garg \\ University of Petroleum \\ \& Energy Studies \\ Dehradun
}

\author{
U. Kedareswarudu \\ University of Petroleum \\ \& Energy Studies \\ Dehradun
}

\begin{abstract}
The use of the information technology in internet GIS, Remote Sensing data and World Wide Web in most of the research works relating geosciences and public is indispensable. As the nodal data banks and public utility are ever evolving along with the technological advancements, it is necessary to have an interoperable linked geospatial infrastructure for surface as well as underground water resources. IWML (India Water-resources Markup Language) is proposed for the monitoring of surface and subsurface water resources data of India. IWML works on Asynchronous java Script and XML and image tiling can improve the performance and response time of Internet GIS application significantly. A framework of IWML and image tiling techniques for Web-based visualization for water resources management is discussed. Functioning of the weboriented water resources information requires a leadership and management support, technical architecture, creating and sharing water resources data, adoption of standards, creation and support of community water data portals and ongoing participation. It serves the multidisciplinary users at diverse applications such as map viewing, query answering, simple mashup's analysis and visualization, customized applications for particular purposes, access to server side modeling, data discovery download for local analysis and developing a common operating dashboard for situational awareness. Water information integration with web GIS, Google, and Satellite will improve the user's ability to integrate, plan, manage and design more holistically the water resources systems.
\end{abstract}

Keywords: Internet GIS, AJAX, Semantic Web Services, Surface and Groundwater resources, Usability

\section{INTRODUCTION}

Many Government Institutes as well as Private Sector Organizations, Academia, Public Enterprises and Social Organizations have been generating data bases and collaborating with each other in need to arrive at a conclusion and share the result. But in emergency, databank of these institutions may not be readily available. Many a time, data acquisition or data generation, analyses or processing and their presentation are to be done individually instead of sharing their data spreads. This is due to improper maintenance of existing information and not channelized. Geographic Information System (GIS) software is for managing the information resources, better understanding and utilization of the data sets. GIS provides sets of tools for collecting, storing, retrieving, transforming and displaying spatial data. GIS provides a framework for integration of information, connect users of data to the sources, provides methods for analysis and collaboration and making them widely accessible and decision support [1].

The use of GIS in water resources has a rich heritage. Some of the key contributions include: base maps of water features of the landscape, digital elevation models delineating watersheds and stream networks, geospatial data preprocessors supporting water resources simulation models, floodplain maps built using automated hydrology and hydraulics and precisely measured land surface elevation [2].

There is every need to integrate water information. GIS can be implemented in multiple patterns that are evolving with new and good technology. New technology like faster computers, high bandwidth internet, huge storage and cloud computing are creating new environments sharing and authoring geographic information. Desktop GIS integrates water resources with widely deployed community tools. Web services enable the user to access a network of distributed services that function as a collective [3].

Monitoring of Surface water and Groundwater resources is dependent on dynamic and static parameters of these water systems as well as meteorological data sets. All this information is large in volume and spatial as well as temporally varying in nature. Judicial usage and timely alert of these resources need to have a common platform (infrastructure) to store the information in a systematic format and link up geospatial and temporal data and not only make available to the end users but also to provide other utilities like processing, synthesis and visualization in 3D and projecting on the spatial and temporal coordinates (Figure1). 
A review of architecture necessary for the realization of the objective is discussed. The web services can make into function through creating three tiers mainly a database tier, an application tier and a client tier. A vision of such an infrastructure, its architecture, utility, functional language and operations is the scope of this paper.

\section{METHODOLOGY}

Internet GIS applications and Web-based mapping tools suffer from the slow response because of the limitation of image sizes and network issues. This new computing paradigm helps to integrate GIS through a services-based architecture that takes advantage of cloud computing and integrates diverse content through Web 2.0 based mashups[4]. Organizations are increasingly creating geo-services, moving from data sharing to creating shared services.

In a simple view, the web consists of three levels of operations which include, the database tier, the application tier and the client tier. Each of these areas provide web services using following five functionalities.

Interoperability: integration of different workstations, software and hardware, data bases and technical tools.

Design of data models: users collectively create data models within a single GIS platform to promote standards and implementation of projects and cater the specific needs of the user.

Distributed services: dynamically integrated and multiply distributed services by linking the users through portals and web gateways.

Expansion and visualization of web services: adds new dimensions and services for the analyses of geospatial and temporal data bases. The users can visualise and analyse their data sets.

Accessibility: the users capture the data bases and models of analyses easily and can display the same in their handsets and mobile phones [1].

This creates a new geospatial infrastructure supporting a large community of users and applications - linking government, business, citizens and education. This supports open access and transparency of information, and better collaboration. New technology like faster computers, high bandwidth internet, huge storage and cloud computing are creating new environments for sharing geographic information. Desktop and web GIS will continue to play a key role.

\section{SERVICES FRAMEWORK}

The Service Oriented Architecture (SOA) is popular as web services implementation model. The SOA is realized by a "publish-bind-find" pattern, in which the service-provider "publishes" a service in the catalog, then the service-client "finds" the necessary service and at the same time service provider "binds" the service with the service-client (Figure 2) [5].

In SOA, a single service cannot perform a complete job. For this the client needs a "service composition". As a part of standard for web services, World Wide Web Consortium (W3C) defines a software application identified by a Uniform Resource Locator (URL) whose interfaces are provided for binding and capable of being defined, described and discovered by Extensible Markup Language (XML) which supports direct interaction with any other software applications. Internet based protocols supports XML based applications for binding user required data. The web service can require a set of instructions in any of web support applications like XML. XML code will access data over the web [5]. This SOA specify three layer/tier architecture as shown in Figure 3 [6].

This architecture provides online water resources information at a single location. It provides the information which includes water flow level, level in surface water bodies, ground water levels, and some other related to meteorology department such as different types of reports for water resources and daily reservoir operations. All of these information sources are accessed through some of the web applications.

\subsection{Database Tier}

In any application the back bone is back end. In this process the back end application stores huge amount of spatial data. Any of the relational database (e.g. Oracle, MySQL etc.) is used for spatial data. Mostly Oracle supports spatial data, which can be accessed through Structure Query Language (SQL) [7]. Water data are stored in distributed tabular databases, each having its own output data format. Commonly measured variables/fields are labeled differently from one organization to another organization. These tabular databases independently maintained by organizations are not spatially enabled. In larger water agencies or water boards, the data for different geographic regions are managed independently from one regional office to another local office. For example, the operator of water supply reservoir system maintains daily water levels, inflow, storage and release of water in reservoir. This information is fragmented in many forms at many locations. It is very difficult to link with proper application from one data source to another. Therefore, standardized/normalized database are to be maintained across the water boards. This will provide proper storage of database. 


\subsection{Application Tier}

It includes new technology in the Internet GIS market by implementing AJAX (Asynchronous Java Script and Extensible Markup Language) and image tiling techniques for Web based $3 \mathrm{D}$ visualization [2][8]. AJAX is common for building mashups for enabling user interaction and other technologies focused on specification of data and visual encoding for feeding mapping mashups. It enables communication between the tiers and processes system inputs and outputs. XML is a communication language of the architecture. The data, services and communication messages are defined in XML [9]. Concerning spatial data, it has not been possible to agree on a common data definition over the years. The consensus over a language provides a great degree of reusability. XML language focuses on geographic visualization including annotation of maps and images [9]. AJAX includes a number of modules which are:

1. Parse user selections from web interface. 2. Access and query the database through web server and generate files for use in Google Earth and Flash. 3. Parse any other user queries generated from Google Earth. The process involves any of the scripting languages like Java Script, Perl for parsing user selections, querying the database and generating output. AJAX generates all features including data layers, models and attribute data and the styling of all features. AJAX application acts as a buffer to avoid the start stop nature of interaction on web by introducing an intermediary layer between the user and the server (Figure 4). An AJAX engine is in between user and the server. The browser loads an AJAX engine with java script code and allows user interaction with the application in asynchronous mode [6]. AJAX engine works on the principle that it sends user's requests to the web server to retrieve only the data needed by the request.

So the total numbers of images or data interchanged between the client browser and web server will be reduced and it becomes faster compared to other applications.
The tiled images are to improve the application performance by allowing the application to process an image region within a number of tiles without bringing the entire image into memory. This image tiling method will provide fast performance in a digital form. The large image will be broken down to smaller tiles with different resolutions in different files. Through AJAX and image tiling method faster performance is achieved [2].

\subsection{Client Tier}

The user interacts with the application software through user interface. As interfaces are medium for communication with distributed information spread across the web thus their designing is crucial for Internet GIS and other web based applications. The usual process is that user hit query, the application tier access the database and answer user request. These information displays need to meet guidelines of usability and accessibility of W3C. In this regard, impetus from Human Computer Interaction is crucial for designing and developing interfaces and geospatial web services.

\section{CONCLUSIONS}

There is a need to integrate Indian water resources information. Internet GIS provides a platform to integrate the available technology with users and data sources. It is necessary to store the data by all the public domains in specified formats and update the information timely. Web services have to provide convenient architecture with sophisticated tools which will give situational awareness and local analyses and presentation. It is necessary to have an interoperable linked geospatial infrastructure for surface and subsurface water resources data of India. Vision of such architecture, IWML, is proposed.

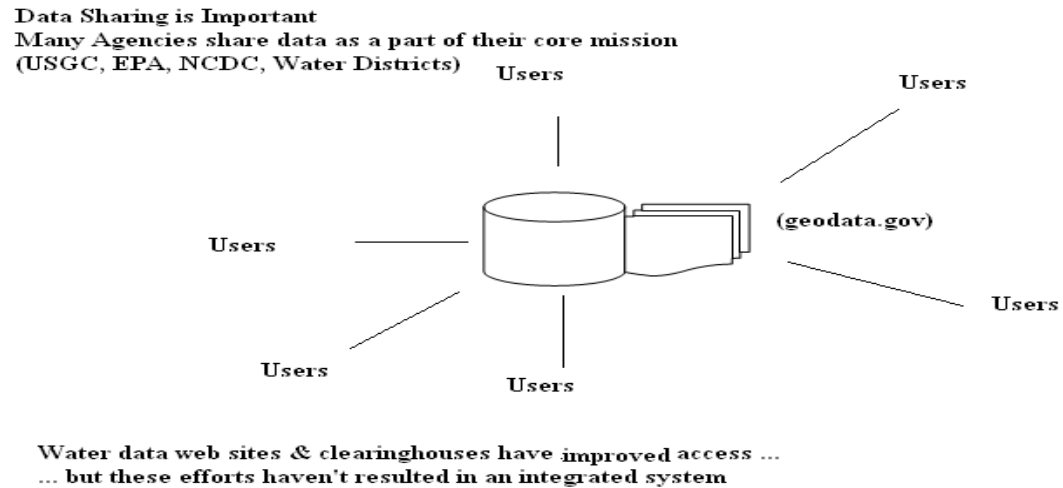

... but these efforts haven't resulted in an integrated system

Figure 1: Sharing water data through the Internet

[Dangermond, J. and Maidment, D. 2010] 


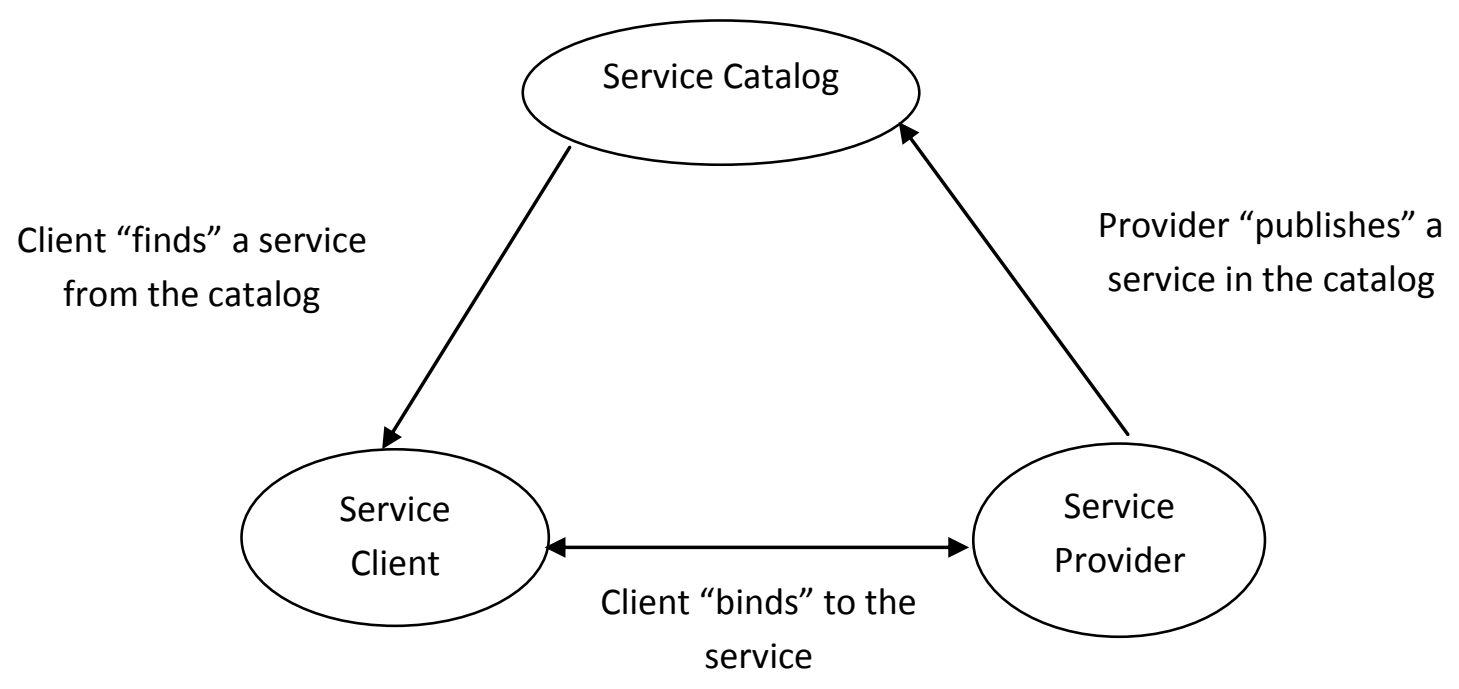

Figure 2: The vision of service oriented architecture (SOA)

[Cetin, C., et al. 2010]

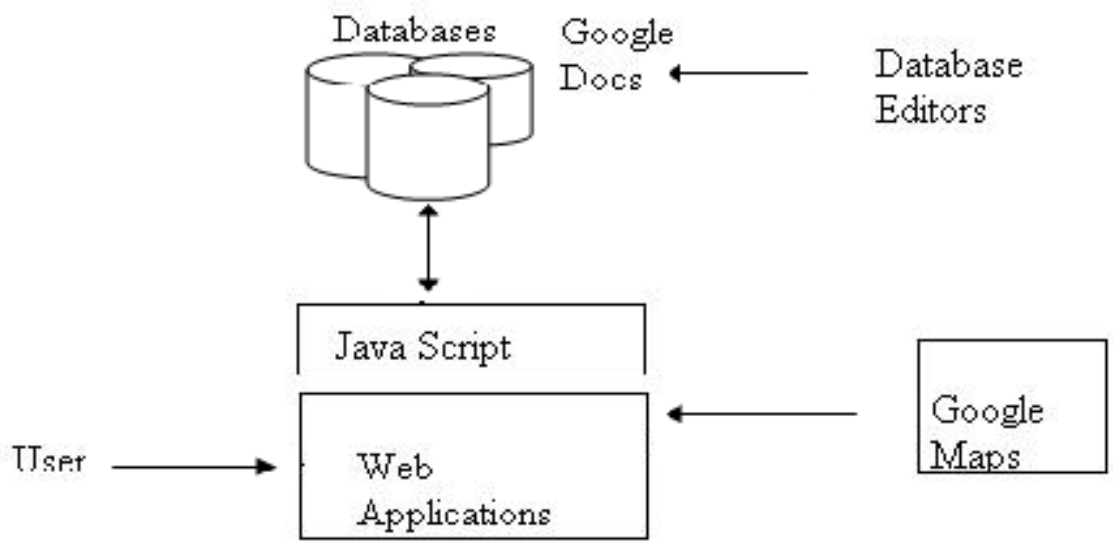

Figure 3: Three tier architecture 


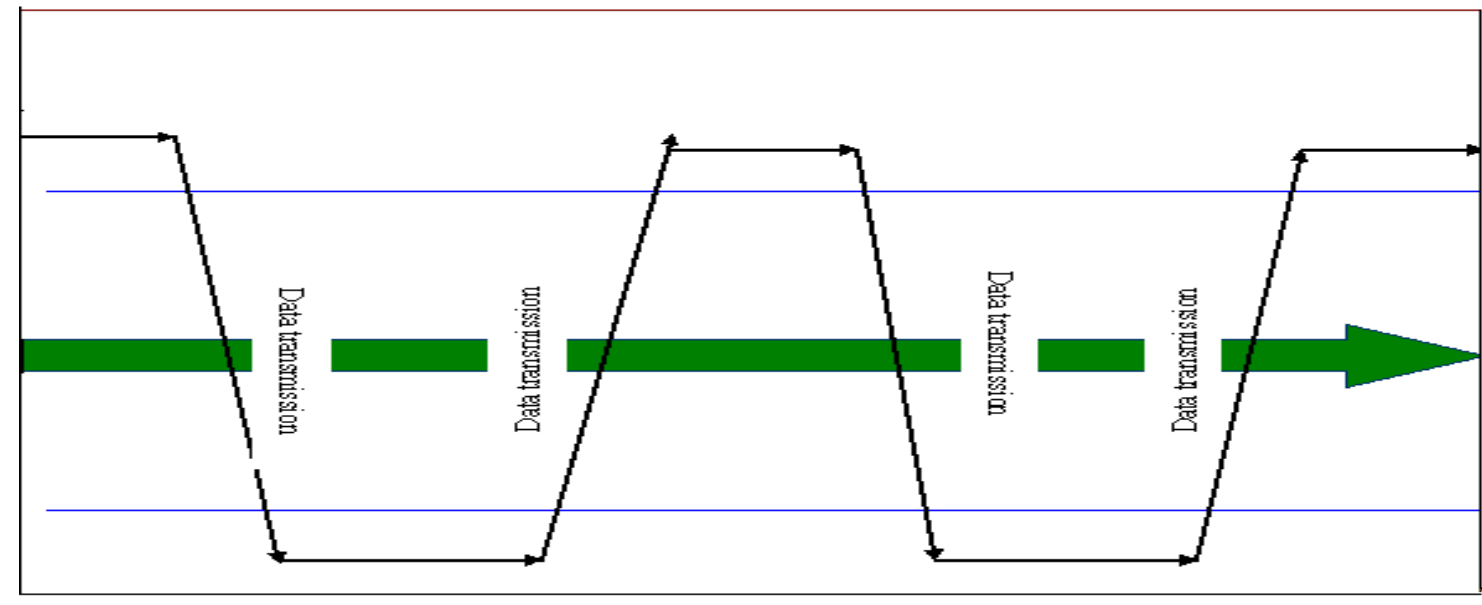

Classic Web Application Model (Synchronous)

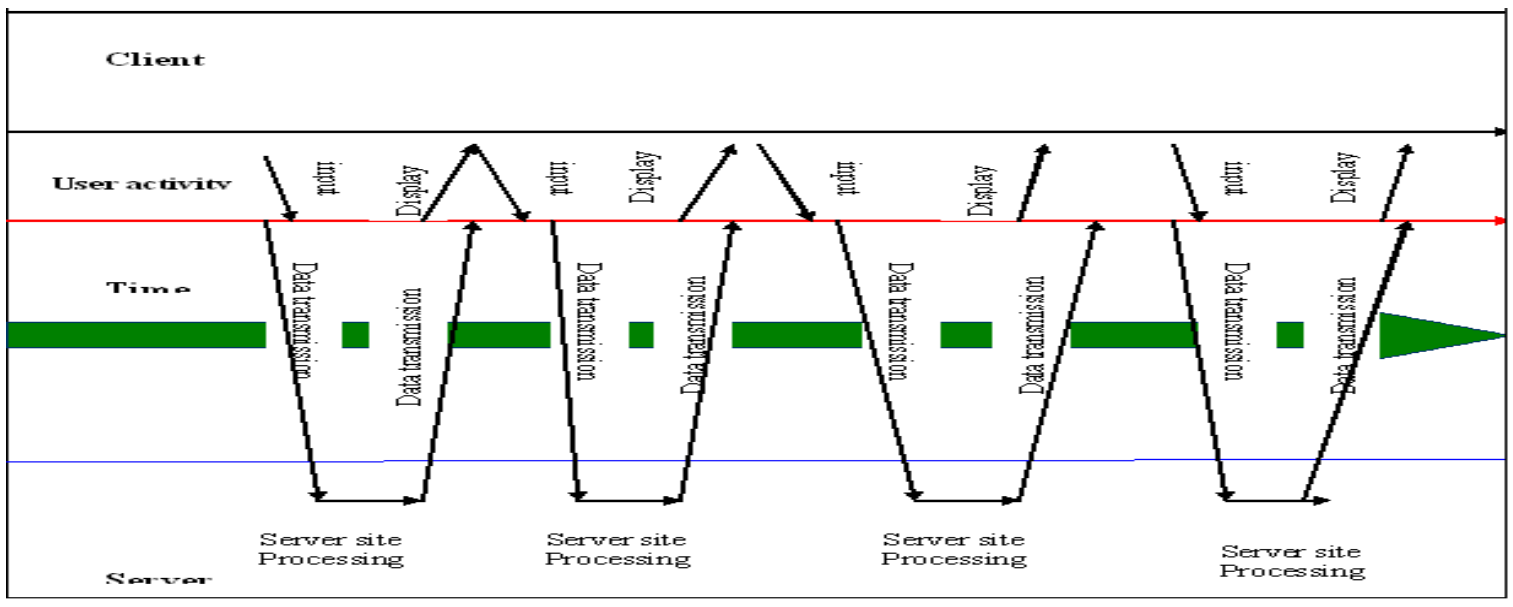

Figure 4: AJAX web application model (Asynchronous)

[Tsou, M. H. 2005]

\section{ACKNOWLEDGMENTS}

The summery of the paper was presented in a National Seminar at Wadia Institute of Himalayan Geology, Dehradun organized by Central Ground Water Board, Dehradun. The authors are thankful to Chancellor, UPES and Director, WIHG for giving encouragement to carry out this work and providing the facilities.

\section{REFERENCES}

[1] Mauree, V. 2010. ICT as an Enabler for Smart Water Management. ITU-T Technology Watch Report, ITU Telecommunication Standardization Bureau, pp. 23.

[2] Tsou, M.H. 2005. Recent developments in Internet GIS. GISdevelopment.net, pp. 6.

[3] Dangermond, J. and Maidment, D. 2010. Integrating Water Resources Information using GIS and the WEB. AWRA 2010 Spring Specialty Conference Orlando, Florida, pp. 110.

[4] Granell, C., Diaz, L., and Gould, M. 2008. Geospatial Web service integration and Mashups for water resource applications. The International archives of the photogrammetry, Remote sensing and spatial information sciences, Vol. XXXVII part B4 pp. 661-666.

[5] Cetin, C., Deniztan, U., Halil, A., and Gulten, K. 2010. Semantic Web Services for implementing National Spatial data infrastructures. Scientific Research and Essays, Vol.5 (7), pp. 685-692.

[6] Henry, A. 2009. Using Google Earth for Internet GIS. M.Sc. Dissertation. Institute of Geography, School of GeoSciences, University of Edinburgh, pp. 24.

[7] Price, J. 2007. Oracle Database 11g SQL, Oracle Press, pp. 690.

[8] Deeprasertkul, P. and Chitradon, R. 2009. Time Dimension on the Internet GIS Application. Proceedings of MAP Asia - Connecting Geospatial Communities-Creating New Dimensions, Singapore.

[9] Brett, M. 2001. Java \& XML, 2nd Edition, O'Reilly, pp. 428. 